\title{
Cervical and scapular instability in subjects with temporomandibular disorder
}

\author{
Maryllian de Albuquerque Vieira(1,2); Maria das Graças Rodrigues de Araújo(1,2); Gabriel Barreto Antonino ${ }^{(3)}$; \\ Angélica da Silva Tenório ${ }^{(1,2)}$; Maria das Graças Paivaa ${ }^{(1,2)}$; Ana Paula de Lima Ferreira ${ }^{(1,2)}$
}

\begin{abstract}
Background: Temporomandibular disorder (TMD) is a set of disorders involving the masticatory muscles, the temporomandibular joint (TMJ) and/or the associated structures. Objectives: To evaluate the occurrence of cervical and scapular instability in subjects with TMD. Methods: A total of 22 patients participated in the study, being 11 of them with TMD, selected using the RDC/TMD criteria, and 11 in the control group. The stabilization capacity of the neck muscles was evaluated through Stabilizer ${ }^{\mathrm{TM}}$ and the muscles of the shoulder girdle through specific tests. Cervical mobility data from both groups were provided using the accelerometer while for cervical disability was used the Neck Disability Index (NDI) questionnaire. Results: Cervical instability was higher in the TMD group (20.36 \pm 3.2$)$ than in the control group ( $28.54 \pm 0.8)$, revealing significant difference $(p=0.03)$. The highest percentages of scapular stabilization tests were found in subjects with TMD, ( $n=9 ; 81.81 \%)$ when compared with control subjects ( $n=5 ; 45.45 \%)$. The NDI results showed that the TMD group presented mild cervical incapacity $(11.18 \pm 2)$ and the control presented no disability $(2.27 \pm 0.4 ; p=0.001)$. Conclusion: Cervical disability, and cervical and scapular instability were more frequent in subjects with TMD.
\end{abstract}

Keywords: Temporomandibular Disorder; Neck; Joint Instability; Scapula.

\section{INTRODUCTION}

The temporomandibular disorder (TMD) is a set of disorders involving the masticatory muscles, the temporomandibular joint (TMJ) and/or the associated structures $^{(1,2)}$. Symptoms of this disease include decreased range of motion of the jaw, pain in masticatory and TMJ muscles, generalized myofascial pain, and deviation of the mandible during mouth opening ${ }^{(3,4)}$.

Anatomical proximity, neuronal interconnections, and trigeminal convergence inputs in cervical areas suggest an association between the TMD and the cervical disorders ${ }^{(5)}$. The clinical interconnection between masticatory and cervical structures may justify the fact that subjects with masticatory myofascial pain have greater cervical incapacity, which may lead to increased regional muscle sensitivity ${ }^{(5)}$.

Jull et al. $(2008)^{(6)}$ showed that the reduction of activation of the deep cervical muscles, the increased superficial activity of the sternocleidomastoid and anterior scalene muscles, the changes in early activation of deep stabilizing muscles, the reduced ability to relax the cervical muscles, and the prolonged muscle activity after voluntary contraction, can compromise the control of the cervical spine, leading and, consequently, leads to pain and dysfunction.

It is known that cervical pain and anterior head posture have a deleterious effect on static and dynamic postural control $^{(7)}$ and that the presence of trigger points may alter the electromyographic activity of cervical muscles ${ }^{(8)}$. Besides, it has been found that patients with TMD tend to have an increased electromyographic activity of the superficial cervical muscles compared to the control group. This increase is justified by a possible association between cervical spine disorders in patients with $\mathrm{TMD}^{(9)}$.

There are few studies that show the relationship between TMD and scapular disorders. Vierola et al. (2012) confirmed the relationship between chest pain, tension to muscle palpation of neck and shoulder, and headache with clinical signs of TMD in children ${ }^{(10)}$. A systematic review of Chaves et al. (2014) showed that there is scapular and cervical misalignment in individuals with $\mathrm{TMD}^{(11)}$.

\footnotetext{
Corresponding Author: Ana Paula de Lima Ferreira. Address: Av. Jornalista Anibal Fernandes, S/N. Cidade Universitária, Recife, PE, Brazil. CEP: 50740-521. Department of Physiotherapy/CCS/UFPE. Telephone: +55 81 982017776. E-mail: apllima@yahoo.com.br
}

1Department of Physiotherapy of Universidade Federal de Pernambuco, (UFPE), Recife (PE), Brazil.

Full list of author information is available at the end of the article.

Financial support: The authors declare that there was no financial support.

Submission date 08 August 2018; Acceptance date 25 September 2018; Publication 11 October 2018 
Due to the lack of studies on this topic, this study aimed to verify the occurrence of cervical and scapular instability in subjects with temporomandibular dysfunction.

\section{METHODS}

This was a cross-sectional, descriptive and observational study. The sample was constituted by convenience with 22 subjects distributed in 02 groups. Group 1 with patients with a diagnosis of muscular TMD and group 2 called control. Subjects in the age group of 18 to 45 years, with chronic pain (painful sensation with at least 3 months of persistence) consequent to the existence of myofascial pain with or without limited mouth opening, were included in the study. Patients with a history of face trauma, rheumatic and orthopedic pathologies, surgical procedures performed in the craniocervical region and neurological diseases, as well as using medications and/or orthodontic appliance at the time of data collection were excluded.

It is noteworthy that for the diagnosis of TMD was used the revised version of Research Diagnostic Criteria - RDC ${ }^{(12-14)}$. The volunteers were assessed at the Laboratory of Kinesiotherapy and Manual Therapeutic Resources (LACIRTEM) in the Department of Physiotherapy of the Universidade Federal de Pernambuco. Before the data collection, the volunteers were clarified about the purpose of the research and signed the Informed Consent Form. The study complied with Resolution 466/12 of the National Health Council, which deals with the Code of Ethics for research on human beings and was approved by the Human Research Ethics Committee of the UFPE with protocol number 2.005.880. This study was approved by the Ethics Committee of Universidade Federal do Pernambuco with protocol number: 2.005.880.

The Cervical Instability Assessment was performed by a Stabilizer $^{\mathrm{TM}}$ (pressure biofeedback unit, Chattanooga Group). This device was folded in three and placed on the cervical spine and inflated up to $20 \mathrm{mmHg}$ (Figure 1). The patient was instructed to perform a slight nod and increase cuff pressure to $22 \mathrm{mmHg}$, maintaining constant pressure. If the patient was able to maintain the position with minimal superficial

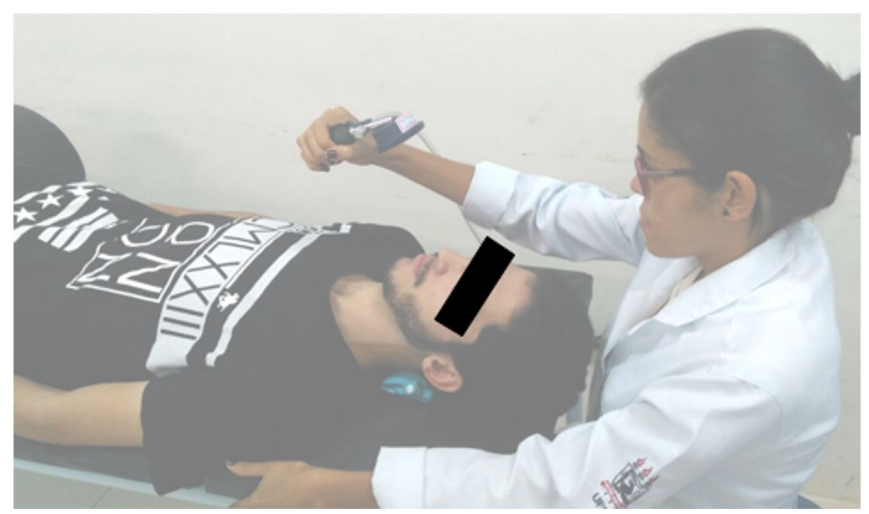

Figure 1. Evaluation with Stabilizer ${ }^{\mathrm{TM}}$. muscular activity, he/she would relax and repeat the flexion, increasing the pressure to $24 \mathrm{mmHg}$. This activation was gradually repeated up to $30 \mathrm{mmHg}$ (total increase of $10 \mathrm{mmHg}$ ). The final pressure was one that the patient was able to keep stable. Muscle fatigue resistance (tonic or maintenance capacity) of the deep neck flexors was measured by the number of contractions maintained for 10 seconds (up to a maximum of 10) at the final pressure.

Jull et al. ${ }^{(15)}$ reported that controlled execution of high cervical flexion increases the pressure in the Stabilizer ${ }^{\mathrm{TM}}$ up to $30 \mathrm{mmHg}$ and that the test-retest reliability of the craniocervial flexion test (conducted in 50 asymptomatic individuals and within one week between the tests) was an intraclass correlation coefficient (ICC) of 0.81 for the activation score and 0.93 for performance indicator.

Scapular instability was assessed through the contraction maintained by the scapular stabilizers: upper trapezius, lower trapezius and serratus anterior. The upper trapezius was tested by raising the shoulder for 5 seconds and the evaluator felt the contraction of this muscle ${ }^{(16)}$. As well as the inferior trapezius, testing the patient's ability to keep the scapula abducted and depressed for 10 seconds while the evaluator felt muscle contraction. The anterior serratus was evaluated in the positions of semi-recumbent support and support on the ground, observing the scapular retraction and elevation of the medial region, indicating muscular inability. The presence of scapular instability was also tested through a basic stabilization of the cervical musculature with progressive loading of the upper limbs. The subject in ventral decubitus and with the forehead on the table performed a lateral rotation of the shoulder and scapular adduction, keeping the arms alongside the body. After that, with the arms in 90/90, performed horizontal abduction and scapular adduction. Then, the shoulder was elevated in full range of motion, progressing to lateral rotation with shoulders in $90^{\circ}$ and elbows extended, horizontal abduction and scapular adduction ${ }^{(17)}$.

The evaluation of the amplitudes of cervical movements was performed with an inertial sensor, without triaxial wire and with $40 \times 45 \times 20 \mathrm{~mm}$ of dimension, Wiva ${ }^{\circledR}$ (Wiva Science Walk). The sensor was positioned in the center of the participant's forehead, who was in the sitting position with hips and knees at 90 o and feet resting on the ground. Rotation, inclination, flexion and extension of the head were required while the evaluator stabilized the patient's shoulders (Figure 2). The patient was instructed to do 3 repetitions of each range of motion, considering for analysis the arithmetic mean obtained. The references of normal ADM considered in this study were in agreement with Magee $(2010)^{(18)}$.

To evaluate the cervical function was used the Neck Disability Index (NDI) questionnaire. Its original version was developed by Vernon and Mior ${ }^{(19)}$ been adapted and validated into the Portuguese language by Cook et al. ${ }^{(20)}$. The score consists of the sum of the points, from 0 to 5 , of each of the 10 questions, totaling a maximum of 50 points. The patient is 
considered with no disability when the result varies from 0 to 4 ; with mild disability when the variation is from 5 to 14 ; with moderate disability when the variation is from 15 to 24 ; with severe disability when the variation is from 25 to 34 ; and with complete disability when the variation is from 35 to 50 .

\section{Statistical Analysis}

Statistical analysis was performed using BioEstat 5.0. Variables were tested for data normality using the Shapiro Wilk test and no impediment to the use of parametric tests was found for any of the parameters evaluated. The values were presented in percentage, mean (X) and standard deviation (SD). The intergroup analysis of the variables was performed through unpaired t-test. The Pearson Correlation Coefficient was used to evaluate the interaction between instability and range of motion of the cervical spine. The reference values considered were: $(r) 1$ and -1 with $r>0.8$ to excellent correlation level; between 0.6 and 0.8 to moderate correlation; from 0.3 to 0.5 to weak correlation; and $r<0.3$ to inexistent correlation. Negative values reproduce inversely proportional

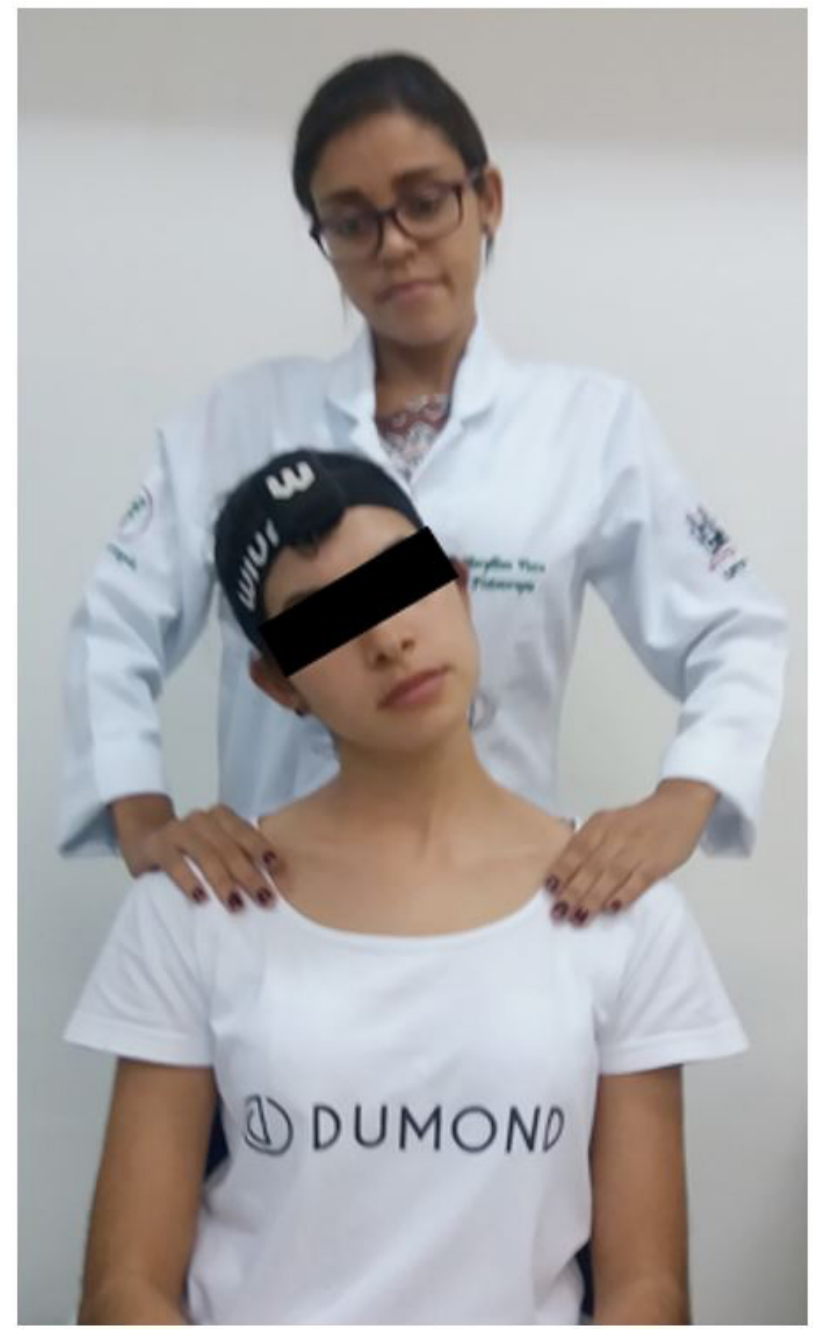

Figure 2. Evaluation of cervical mobility. correlation, while positive values reproduce directly proportional correlation ${ }^{(21)}$. The level of significance for all tests was $5 \%$.

\section{RESULTS}

The sample consisted of 22 subjects, 11 in each group. The majority of the participants were women: $72.72 \%(n=8)$ in the TMD group and $54.54 \%(n=6)$ in the control group. There were no differences between groups regarding age (TMD 25 \pm 1.8 and control 23.6 $\pm 1.4 ; \mathrm{p}=0.471$ ) and body mass index (TMD 23.01 \pm 3.29 and control 23.16 $\pm 3.50 ; p=0.921$ ). Table 1 shows the values related to the presence of cervical and scapular instability of the sample, in which the TMD group and the control group showed, respectively, mean of $20.36 \pm 3.2$ and $28.54 \pm 0.8$ to cervical instability. And 9 of the 11 subjects in the TMD group (81.81\%) presented scapular instability.

The mean results of the NDI were $11.18 \pm 2$ for the TMD group and $2.27 \pm 0.4$ for the control group $(p=0.001)$. The range of motion showed lower ranges in the TMD group for extension $(p=0.02)$. Whereas there was no significant difference for the other movements (table 2).

When analyzing the correlation between cervical instability and cervical range of motion, a weak positive correlation was found only for cervical rotation in the muscle TMD group (Figure 3). There was no correlation for the other range of motion.

\section{DISCUSSION}

The main findings of this study show that subjects with TMD had cervical and scapular instability. In addition, patients with TMD demonstrated greater cervical spine disability

Table 1. Cervical Instability and Scapular Instability between groups.

\begin{tabular}{|c|c|c|c|}
\hline & $\begin{array}{l}\text { TMD } \\
(n=11)\end{array}$ & $\begin{array}{l}\text { CONTROL } \\
(n=11)\end{array}$ & $\begin{array}{c}\text { p-Value } \\
\mathrm{Cl}(95 \%) \text { diff. between mean }\end{array}$ \\
\hline $\begin{array}{c}\text { Cervical } \\
\text { Instability }\end{array}$ & $20.36 \pm 3.2$ & $28.54 \pm 0.8$ & $\begin{array}{c}0.030 * \\
\mathrm{Cl}(-15.65 \text { to }-0.71)-8.18\end{array}$ \\
\hline $\begin{array}{l}\text { Scapular } \\
\text { Instability }\end{array}$ & $9(81.81 \%)$ & $5(45.45 \%)$ & \\
\hline
\end{tabular}

Table 2. Range of motion of the cervical spine (angle) between groups.

\begin{tabular}{cccc}
\hline & TMD $(\mathbf{n}=\mathbf{1 1})$ & CONTROL $(\mathbf{n = 1 1 )}$ & p-Value \\
\hline Flexion & $60.30 \pm 7.8$ & $60.52 \pm 2.95$ & 0.96 \\
Extension & $48.90 \pm 2.9$ & $63.83 \pm 4.07$ & $0.02^{*}$ \\
Inclination R & $39.77 \pm 2.96$ & $40.47 \pm 2.55$ & 0.86 \\
Inclination L & $41.25 \pm 3.11$ & $43.63 \pm 1.84$ & 0.43 \\
Rotation R & $65.11 \pm 3.0$ & $64.11 \pm 2.98$ & 0.46 \\
Rotation L & $65.83 \pm 3.79$ & $64.83 \pm 4.47$ & 0.44 \\
\hline
\end{tabular}

Note: R= Right side; L= Left side; Unpaired t-test; ${ }^{*} \mathrm{p}<0.05$. 


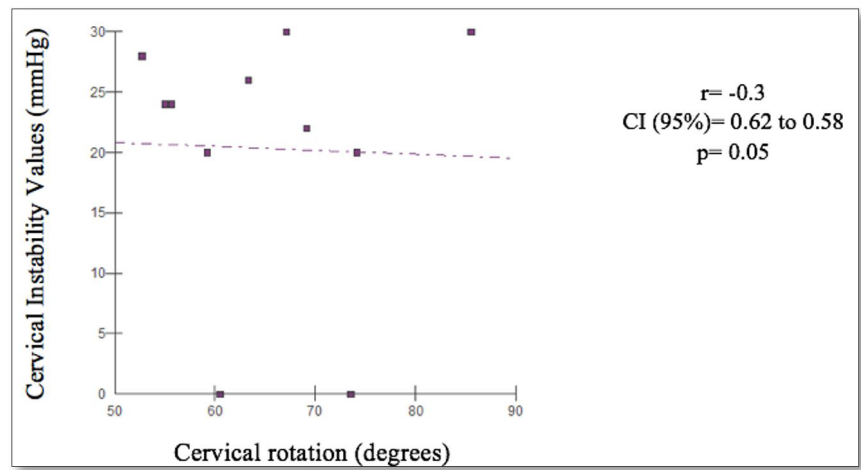

Figure 3. Correlation between Cervical Instability $(\mathrm{mmHg})$ and Cervical Rotation Range (degrees) of the TMD group.

and presence of weak positive correlation between cervical instability and cervical rotation.

The flexor and extensor muscles of the neck must stabilize the head and neck to maintain posture and resist dysfunction. It should be considered that the level of optimal muscle activation depends on factors such as the level of individual ability ${ }^{(22)}$. The neck muscles are considered complex structures with multiple tasks to maintain proper functioning of the cervical spine ${ }^{(23)}$. The cervical stabilization analysis investigated in the present study was carried out to investigate changes in the behavior of these muscles in the presence of TMD.

The presence of cervical instability observed in this study for patients with TMD corroborates the findings of Armijo-Olivo et al. (2012), which showed a strong relation between neck disability and TMD ( $r=0.82)$. The subjects with greater disability in TMJ were more predisposed to have a greater disability of the cervical and vice versa ${ }^{(24)}$.

Cervical instability in patients with TMD was also evidenced by Wiesinger et al. (2009) ${ }^{(25)}$, which indicated frequent comorbidity between these two conditions. These authors argue that cervical instability and TMD may be associated with risk factors or may influence one another. In addition, they showed that the prevalence of fatigue or stiffness, pain, decreased mouth opening and headaches, as well as the general prevalence of TMD and aggravation of its symptoms, increased in a dose-response pattern regarding frequency/severity of cervical pain (neck, shoulder or chest pain) $)^{(25)}$.

The scapular instability of patients with TMD has been sparsely studied. In the present study, this outcome was manifested in 9 of 11 subjects in TMD group (81.81\%). The literature review conducted did not find the existence of studies that related to scapular instability with TMD. However, the postural relationship of the shoulder girdle in patients with TMD has been reported in the literature. Ferão et al. $(2008)^{(26)}$ evaluated the posture of subjects with TMD, noting postural changes of shoulders and head. In a systematic review, Chaves et al. (2014) demonstrated that of the seven studies that verified postural changes in the shoulders in patients with TMD, four of them observed changes in this segment: asymmetric shoulders and abducted scapulae, protrusion and elevation of the shoulders ${ }^{(11)}$, i.e., changes in the shoulder girdle were closely related to the presence of TMD.

Amantéa et al. (2004), in a literature review, showed that subjects with TMD tended to have uneven shoulders ${ }^{(23)}$. According to Farias et al. (2001), the head tends to be tilted to the side of the affected TMJ as the shoulder tends to be protruding or elevated on the same side of the affected $\mathrm{TMJ}^{(27)}$. Although there are no reports of studies on the presence of scapular instability in individuals with TMD, it is believed that biomechanical mechanisms integrate the craniocervical-scapular segment so that there are influences from one segment over the other.

The cervical disability observed in patients with TMD was similar to the findings of Costa et al. ${ }^{(5)}$, in which the symptomatic group presented mild cervical instability while the control group had no disability, with a statistically significant difference between the groups. As well as the study of Silveira et al. ${ }^{(28)}$, which demonstrated that TMJ dysfunction and neck disability were significantly higher in subjects with TMD showing that there is an association between TMD and cervical incapacity.

Armijo-Olivo et al. (2010) were precursors in emphasizing the existence of the relationship between TMD and neck disability, showing that disability is a complex concept, since it involves more than counting only the signs and symptoms of each subject ${ }^{(29)}$. In the present study, the reports collected in the NDI represent the impact of the disease on the patient's daily functions and more accurately reflect how much their complaints affect the abilities to perform their daily activities.

As a contribution, this study draws attention to the importance of observing the implications of cervical and scapular instability in the performance of daily activities of subjects with TMD. The existence of scapular instability in patients with TMD points to the paradigm change in the current therapeutic approach, since in practice, assessments and exercise orientations are usually not performed to the improvement of the shoulder girdle stabilization of these subjects.

\section{CONCLUSIONS}

There was the presence of cervical and scapular instability in subjects with TMD in the studied sample. Therefore, the evaluation of these outcomes is considered important in the physical evaluation intervening in the treatment of patients who present this dysfunction together with cervical and scapular incapacity, and it should focus on both areas, since the improvement of one could have influence on the other.

\section{AUTHORS CONTRIBUTIONS}

MAV: Data collection and study design; MGRA: study design and conception, and analysis and interpretation of data; GBA: Data collection and study design; AST: analysis and interpretation of data, and draft of the article; MGP: statistical analysis and critical review; APLF: was responsible for all aspects of the research 
in order to ensure that issues related to the accuracy and integrity of all parts were properly investigated and resolved. All authors read and approved the final manuscript.

\section{CONFLICT OF INTEREST}

The authors declare that there was no conflict of interest.

\section{AUTHORS DETAILS}

(2)Physiotherapy School, Universidade Federal de Pernambuco (UFPE), Recife (PE), Brazil.(3) Physiotherapy Post-Graduation Program, Universidade Federal de Pernambuco (UFPE), Recife (PE), Brazil.

\section{REFERENCES}

1. Robert L. Gauer, MD, Michael J. Semidey, DMD. Diagnosis and treatment of Temporomandibular disorders. Am Fam Physician. 2015;91(6):378-86.

2. Scheers, Isabelle, et al. "NCBINCBI Logo Skip to main content Skip to navigation Resources How To About NCBI Accesskeys Sign in to NCBI PubMed US National Library of Medicine National Institutes of Health Search database Search term Clear input Advanced Help Result Filters Format: Abstract Send to J Pediatr Gastroenterol Nutr. 2018 May 9." Journal of Pediatric Gastroenterology and Nutrition (2018).

3. Leeuw R. Dor orofacial: guia de avaliação, diagnóstico e tratamento. 4. ed. São Paulo: Quintessence, 2010.

4. Wadhwa S, Kapila S. TMJ Disorders: future innovations in diagnostics and therapeutics. J Dent Educ. 2008; 72(8):930-47.

5. Costa da DR, Lima APF, Pereira TA, Porporatti AL, Conti PC, Costa YM et al. Neck disability is associated with masticatory myofascial pain and regional muscle sensitivity. Arch Oral Biol. 2015;60(5):745-52.

6. Jull GA, Sterling M, Falla D, et al. Whiplash, Headache, and Neck Pain: Research-Based Directions for Physical Therapies. Philadelphia, PA: Elsevier; 2008:41-58.

7. Soares JC, Weber P, Trevisan ML, Mota CB, Rossi AG. Influence of pain on postural control in women with neck pain. Rev Bras Cineantropom Desempenho Hum 2013, 15(3):371-381.

8. Saavedra FJ, Cordeiro MT, Alves JV, Fernandes HM, Reis VM, Alverne DG. The influence of positional release therapy on the myofascial tension of the upper trapezius muscle. Rev Bras Cineantropom Desempenho Hum 2014, 16(2):191-199.

9. Armijo-Olivo S, Silvestre R, Fuentes J, Costa da BR, Gadotti IC, Warren S. et al. Electromyographic Activity of the Cervical Flexor Muscles in Patients With Temporomandibular Disorders While Performing the Craniocervical Flexion Test: A Cross-Sectional Study. Phys Ther. 2011; 91(8): 1184-1197.

10. Vierola A, Suominen AL, Ikavalko T, Lintu N, Lindi V, Lakka HM. et al. Clinical signs of temporomandibular disorders and various pain conditions among children 6 to 8 years of age: the PANIC study. J Orofac Pain. 2012;26(1):172.

11. Chaves TC, Turci AM, Pinheiro CF, Sousa LM, Grossi DB. Static body postural misalignment in individuals with temporomandibular disorders: a systematic review. Braz J Phys Ther. 2014. 18(6):481-501.
12. Schiffman EL, Ohrbach R, Trulove EL, Feng T, Anderson GC, Pan W et al. The research diagnostic criteria for temporomandibular disorders. V: methods used to estabilish and validate revised axis I diagnostic algoritms. J. Orofacial Pain, 2010. 24(1): 63-78.

13. Dworkin SF, Leresche L. Research diagnostic criteria for temporomandibular disorders: review, criteria, examinations and specifications, critique. J Craniomandib Disord, 1992. 6(4):301-355.

14. Pereira FJ. Critérios de Diagnóstico para a pesquisa das disfunções temporomandibulares (RDC/TDM): tradução oficial para Língua Portuguesa. J. Bras. Clin. Odontol. 2004. 8:384-395.

15. Jull G, Barrett C, Magee R, Ho P. Further clinical clarification of the muscle dysfunction in cervical headache. Cephalalgia. 1999; 19(3):179-85.

16. Sforza C, Rosati R, De Meneses M, Musto F, Toma M. EMG analysis of trapezius and masticatory muscles: experimental protocol and data reproducibility. J Oral Rehabil. 2011;38(9):648-54.

17. Kisner C, Colby LA. Exercícios terapêuticos: fundamentos e técnicas. 5.ed. Barueri, SP: Manole, 2009.

18. Magee DJ. Avaliação Musculoesquelética. Ed. Manole, 2010.

19. Vernon H.; Mior S. The Neck Disability Index: a study of reliability and validity. J Manipulative Physiol Ther. 1991;14(7):409-15.

20. Cook C, Richardson JK, Braga L, Menezes A, Soler X, Kume P et al. Crosscultural adaptation and validation of the Brazilian Portuguese version of the Neck Disability Index and Neck Pain and Disability Scale. Spine. 2006;31 (14):1621-7.

21. Dancey C, Reidy J. Estatística sem matemática para psicologia: usando SPSS para Windows. Porto Alegre: Artmed, 2006.

22. García-Orea PG, Heredia JR, Sanotos MS, Grigoletto ME. Acute effects of the use of external instability generating devices on neuromuscular performance. Rev Bras Cineantropom Desempenho Hum 2016, 18(6):722730.

23. Amatéa DV, Novaes AP, Campolongo GD, Barros de TP. A importância da avaliação postural no paciente com disfunção da articulação temporomandibular. Acta Ortop. Bra. 2004;12(3).

24. Armijo-Olivo S, Magee D. Cervical Musculoskeletal Impairments and Temporomandibular Disorders. J Oral Maxillofac Res. 2012; 3(4):1.

25. Wiesinger B, Malker H, Englund E, Wänman A. Does a dose-response relation exist between spinal pain and temporomandibular disorders? BMC Musculoskelet Disord. 2009;10:28.

26. Ferão MIB, Traebert J. Prevalence of temporomandibular dysfunction in patients with cervical pain under physiotherapy treatment. Fisioter. Mov. 2008;21(4):63-70.

27. Farias ACR, Alves VCR, Gandelman H. Estudo da relação entre disfunção da articulação temporomandibular e as alterações posturais. Rev Odontol UNICID. 2001;13(2):125 - 133

28. Silveira A, Gadotti IC, Armijo-Olivo S, Biasotto-Gonzalez DA, Magee D. Jaw Dysfunction Is Associated with Neck Disability and Muscle Tenderness in Subjects with and without Chronic Temporomandibular Disorders. Biomed Res Int. 2015:512-792.

29. Armijo-Olivo S, Fuentes J, Major PW, Warren S, Thie NMR, Magee DJ. "The association between neck disability and jaw disability," J. of Oral Rehabilitation. 2010; 37(9): 670-679. 